\title{
Propriedades Térmicas de Polímeros por Métodos Transientes de Troca de Calor
}

\author{
Wilson N. dos Santos, Rinaldo Gregório F \\ UFSCar, DEMa \\ Paul Mummery, Andrew Wallwork \\ University of Manchester, Manchester Materials Science Centre, UK
}

\begin{abstract}
Resumo: A determinação precisa das propriedades térmicas de um polímero é fundamental em muitos processos e projetos de engenharia. A difusividade térmica, em particular, é importante em problemas envolvendo estado não-estacionário, como no aquecimento e resfriamento de um polímero. Em processamento é uma propriedade fundamental no processo de moldagem por injeção, para a determinação do tempo de ciclo de moldagem. Neste trabalho, a técnica de pulso de energia, normalmente aplicada para materiais cerâmicos e metálicos, é empregada na determinação da difusividade térmica de materiais poliméricos. As amostras foram preparadas em forma de discos, tendo aproximadamente $1 \mathrm{~cm}$ de diâmetro e 0,3 a $1 \mathrm{~mm}$ de espessura. Os resultados experimentais obtidos foram comparados com dados existentes na literatura, e com aqueles obtidos pela técnica de fio quente. A concordância entre esses resultados e a reprodutibilidade das medidas mostrou a viabilidade da utilização desta técnica experimental para materiais poliméricos.
\end{abstract}

Palavras-chave: Difusividade térmica, pulso de energia, polímero.

\section{Thermal Properties of Polymers by Non-Steady State Methods}

Abstract: The accurate determination of the thermal properties of a polymer is of fundamental importance in many processes and engineering projects. Specifically, the thermal diffusivity is an important property in the case of non-steady state problems, which occur during the heating and cooling of a polymer. In the extrusion process, the knowledge of the thermal properties is crucial in order to describe and analyse the extrusion process. In this work, the thermal diffusivity of some polymeric materials is determined by using the thermal diffusivity flash technique. The samples are prepared in the shape of discs, $1 \mathrm{~cm}$ in diameter and 0.3 to $1 \mathrm{~mm}$ thickness. The experimental results obtained are compared with those found in the literature. The reproducibility of the data is very good and they are in excellent agreement with the results obtained by the hot wire method.

Keywords: Thermal diffusivity, laser flash technique, polymer.

\section{Introdução}

Durante o processo de extrusão o polímero experimenta uma complicada história térmica ${ }^{[1]}$. No trajeto inicial da extrusora, as partículas sólidas do polímero são aquecidas até o ponto de fusão. Na região intermediária, a temperatura do polímero fundido é aumentada até um nível considerável acima do ponto de fusão, enquanto as partículas sólidas remanescentes continuam sendo aquecidas até a sua fusão. Na região final da extrusora, o polímero fundido tem que alcançar um estado termicamente homogêneo. Quando o material deixa a extrusora ele é, então, resfriado normalmente até a temperatura ambiente. Pode-se concluir, portanto, que o conhecimento das propriedades térmicas de polímeros é crucial na descrição e análise do processo de extrusão.

A condutividade térmica dos polímeros é bem baixa, quando comparada com a condutividade dos materiais metáli- cos, e de alguns materiais cerâmicos. Do ponto de vista de processamento, a baixa condutividade térmica cria alguns problemas reais ${ }^{[1]}$ : ela limita a taxa com a qual o polímero pode ser aquecido e plastificado. No resfriamento, a baixa condutividade pode provocar não uniformidade de temperatura e encolhimento. Isto pode resultar em tensões de congelamento, deformação do extrudado, delaminação, vazios de moldado, etc. A condutividade térmica de um material plástico sólido depende da cristalinidade do material, e portanto da história térmica prévia. Essa propriedade é também afetada pela anisotropia da amostra, e quando esta existe, é recomendado que se façam medidas adicionais apropriadas ${ }^{[2]}$.

O calor específico de polímeros amorfos aumenta linearmente com a temperatura, abaixo e acima da temperatura de transição vítrea ( $\mathrm{Tg}$ ), sendo que ao redor desta temperatura ocorre uma variação brusca dessa propriedade. No caso de polímeros semi-cristalinos essa variação próxima a Tg é menos pronunciada, ocorrendo, entretanto, um

Autor para correspondência: Wilson N. dos Santos, UFSCar, DEMa, Rod. Washington Luiz, Km 235, CP 676, CEP: 13565-905, São Carlos, SP. E-mail:dwns@power.ufscar.br 
máximo distinto no ponto de fusão cristalino. Para uma estrutura cristalina perfeitamente uniforme o calor específico é teoricamente infinito no ponto de fusão. Desde que este não é o caso de polímeros semi-cristalinos, o calor específico destes materiais exibe no ponto de fusão um pico com uma certa largura. Quanto mais estreito for esse pico, mais uniforme é a morfologia cristalina (os cristais apresentam lamelas de espessuras aproximadamente iguais). Acima do ponto de fusão o calor específico aumenta suavemente com a temperatura.

A difusividade térmica é importante em problemas envolvendo estado não-estacionário, o que ocorre durante o aquecimento e resfriamento de um polímero. Em processamento é uma propriedade fundamental no processo de moldagem por injeção, para a determinação do tempo de ciclo de moldagem. Na análise da maioria dos problemas de extrusão ela é considerada constante, embora na realidade ela depende da pressão, temperatura e orientação molecular.

Para o caso de polímeros que são utilizados como isolantes térmicos, a determinação precisa dessas propriedades é importante na avaliação do desempenho desses materiais, e na utilização racional e economia de energia, que são demandas importantes de nossa época.

Assim sendo, pode-se concluir que o conhecimento dessas propriedades é fundamental em muitos processos e projetos de engenharia de polímeros.

Hoje são conhecidos vários métodos para a determinação e estudo das propriedades térmicas de materiais. Entre eles estão os chamados métodos transientes ou não estacionários.

Os métodos transientes de troca de calor são aqueles onde a propriedade térmica é medida segundo um regime transiente de troca de calor. Recentemente, Santos e Gregório ${ }^{[3]}$ utilizaram o método do fio quente na determinação das propriedades térmicas de alguns polímeros. É um método não estacionário, com o qual é possível, a partir do mesmo transiente térmico experimental, determinar simultaneamente a condutividade térmica, o calor específico, e a difusividade térmica do material. Nesta técnica, as amostras são preparadas em forma de paralelepípedos retangulares com as dimensões aproximadas de (230x80x30)mm. Entretanto, por razões tecnológicas, ou econômicas, ou às vezes por ambas, nem sempre é possível a preparação de amostras com as dimensões requeridas por essa técnica experimental, o que inviabiliza a utilização do método de fio quente para muitos materiais poliméricos. Neste trabalho, é utilizada a técnica de pulso de energia na determinação da difusividade térmica de polímeros. Neste método, também não estacionário, as amostras são preparadas em forma de discos, tendo aproximadamente $1 \mathrm{~cm}$ de diâmetro, e 0,3 a $1 \mathrm{~mm}$ de espessura. Os resultados experimentais obtidos foram, então analisados e comparados com os aqueles existentes na literatura, e em alguns casos, com os determinados pela técnica de fio quente.

\section{0 método de pulso de energia}

A técnica de pulso de energia, utilizada neste trabalho, foi introduzida em 1961 por Parker et al ${ }^{[4]}$. Nesta técnica, um pulso de energia radiante e de curta duração incide na face frontal de uma amostra em forma de disco, e o transiente de temperatura na face posterior é registrado.

$\mathrm{Na}$ ausência de perdas de calor, o acréscimo de temperatura na face posterior da amostra é dado por:

$$
\mathrm{V}=1+2 \sum_{\mathrm{n}=1}^{\infty}(-1)^{\mathrm{n}} \exp \left(-\mathrm{n}^{2} \omega^{2}\right)
$$

onde: $\mathrm{V}=\mathrm{T} / \mathrm{T}_{\mathrm{m}}=$ acréscimo adimensional de temperatura na face posterior, $\mathrm{T}=$ acréscimo de temperatura na face posterior, $\mathrm{T}_{\mathrm{m}}=\mathrm{Q} / \mathrm{\rho cL}=$ máximo acréscimo de temperatura na face posterior, $\mathrm{Q}=$ energia do feixe incidente, $\rho=$ densidade, $\mathrm{c}=$ calor específico, $\mathrm{L}=$ espessura da amostra e $\omega=\pi^{2} \alpha \mathrm{\alpha t} / \mathrm{L}^{2}=$ parâmetro adimensional, onde $\alpha$ é a difusividade térmica e té o tempo decorrido a partir do disparo do pulso de energia.

A figura 1 mostra o transiente de temperatura dado pela equação 1.

Parker et al sugeriram duas maneiras distintas para se determinar a difusividade térmica a partir da equação 1 e figura 1.

Quando V é igual a $0,5, \omega$ é igual a 1,38 , e assim, obtémse:

$$
\alpha=1,38 \mathrm{~L}^{2} / \pi^{2} \mathrm{t}_{1 / 2}
$$

onde $\mathrm{t}_{1 / 2}$ é o tempo necessário para que o aumento de temperatura na face posterior da amostra atinja a metade do seu valor máximo.

Uma outra relação útil pode ser obtida de acordo com a segunda sugestão dada por Parker et al. Quando o prolongamento da porção linear da curva da figura 1 intercepta o eixo $\omega$ tem-se que $\omega_{\mathrm{x}}$ é igual a 0,48 obtendo-se, então, a equação:

$$
\alpha=0,48 \mathrm{~L}^{2} / \pi^{2} \mathrm{t}_{\mathrm{x}}
$$

onde $t_{x}$ é o tempo correspondente à interseção do prolongamento da porção linear da curva com o eixo $\omega$.

A condutividade térmica do material é então calculada a partir da difusividade térmica determinada experimentalmente, com o auxílio da equação:

$$
\kappa=\rho c \alpha
$$

onde: $\alpha=$ difusividade térmica $\left(\mathrm{m}^{2} / \mathrm{s}\right), \mathrm{k}=$ condutividade térmica $(\mathrm{W} / \mathrm{mK}), \rho=$ densidade $\left(\mathrm{Kg} / \mathrm{m}^{3}\right), \mathrm{c}=$ calor específico

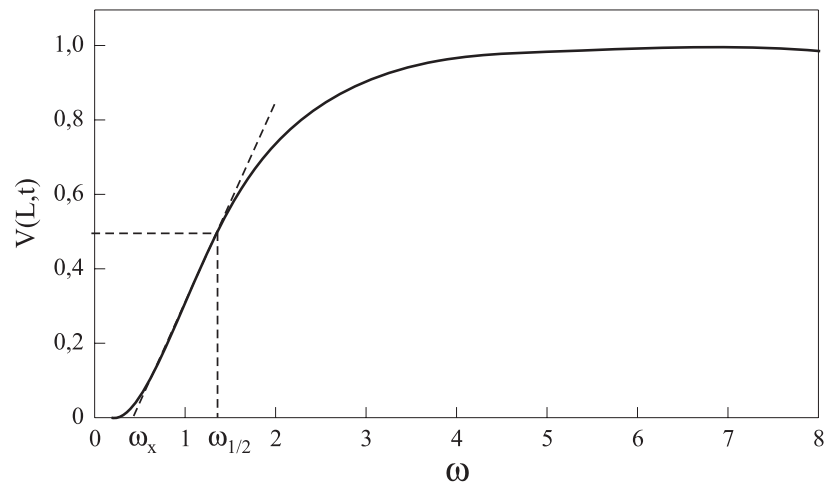

Figura 1. Transiente de temperatura adimensional. 
( $\mathrm{J} / \mathrm{Kg} \mathrm{K})$. Neste caso, o calor específico e a densidade da amostra devem ser previamente conhecidos.

Entretanto, a condutividade térmica pode ser determinada sem o conhecimento prévio do calor específico e da densidade da amostra. Quando se utiliza a equação $T_{m}=Q / p c L$, a energia $Q$ incidente na face frontal de uma amostra padrão pode ser calculada medindo-se a espessura e o aumento de temperatura na face posterior dessa amostra padrão, cujo calor específico e densidade devem ser conhecidos neste caso. Se as condições experimentais são mantidas, isto é, se a energia da fonte térmica e a quantidade de energia que atinge a face frontal da amostra são as mesmas, a quantidade de energia $Q$ absorvida por qualquer face frontal é constante. Assim, a partir da equação (4) obtém-se:

$$
\mathrm{k}=\alpha \mathrm{Q} / \mathrm{L} \mathrm{T}_{\mathrm{m}}
$$

através da qual é possível determinar a condutividade térmica de uma amostra, sem o conhecimento do calor específico e da densidade da amostra.

Tempo finito de pulso e perdas de calor são fatores de desvios das condições de contorno. Clark e Taylor ${ }^{[5]}$ concluíram que o efeito de tempo finito de pulso pode ser desprezível, preparando-se amostras com uma espessura adequada. Todavia, as perdas de calor são impossíveis de serem evitadas em altas temperaturas. Vários trabalhos analisando este efeito foram publicados, e as correspondentes correções devido a essas perdas de calor podem ser feitas usando as expressões de correção propostas.

O equipamento utilizado neste trabalho foi desenvolvido por Taylor ${ }^{[6]}$, na Universidade de Manchester/UMIST, Inglaterra. Os componentes básicos desse equipamento são: uma fonte pulsada de energia, porta-amostra e sistema de aquecimento, sistema de detecção e amplificação do sinal e sistema de análise e processamento dos dados. A figura 2 mostra um diagrama esquemático deste arranjo experimental.

A fonte pulsada de energia é um laser $\mathrm{Nd}$ : glass $(\lambda=1,067 \mu \mathrm{m})$, sendo de $16 \mathrm{~mm}$ o diâmetro do feixe. A energia dissipada pode variar de 5 a $95 \mathrm{~J}$, e o tempo de dissipação do pulso é nominalmente $0,6 \mathrm{~ms}$.

O porta-amostras tem a forma de um disco e é ajustado

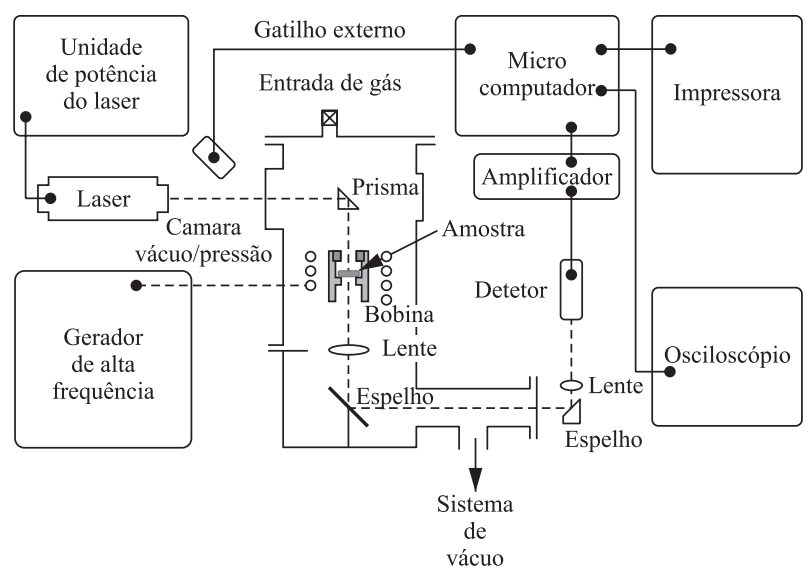

Figura 2. Diagrama esquemático do arranjo experimental utilizado na técnica de pulso de energia. dentro de um susceptor. Uma tampa, com o mesmo diâmetro da amostra é também ajustada na parte superior do susceptor, e tem a finalidade de cortar o excesso do feixe de laser. Todos os componentes são feitos de grafita. Antes de ser colocada no porta-amostras, ambas as faces da amostra são cobertas por uma fina camada de grafita coloidal, para melhorar a sua emissividade.

O aquecimento é proporcionado por um sistema de indução e permite medidas desde a temperatura ambiente até $3000 \mathrm{~K}$. A temperatura da amostra é monitorada por um termopar, ou por um pirômetro ótico, dependendo do intervalo de temperatura das medidas. O susceptor em forma de um cilindro é colocado dentro do sistema de indução, que é montado dentro de uma câmara de vácuo ou pressão, tornando assim, possível, medidas em diferentes atmosferas ou vácuo.

A radiação emitida pela face oposta da amostra é coletada dentro deste vaso e transmitida a um sistema de detecção externo a esta câmara por um sistema ótico. $\mathrm{O}$ detetor de radiação utilizado é de InSb, sensível a 5,5 $\mu \mathrm{m}$ e com um tempo de resposta de $1,5 \mu$ s. O sinal de saída do detetor é amplificado por um amplificador adequado, armazenado e processado por um computador, via um conversor analógicodigital. O sistema de aquisição e processamento de dados é totalmente automatizado, e um código de computação foi especialmente desenvolvido para este objetivo.

\section{Experimental}

As amostras foram preparadas em forma de discos, tendo aproximadamente $1 \mathrm{~cm}$ de diâmetro por 0,3 a $1 \mathrm{~mm}$ de espessura. Dois processos foram utilizados na preparação dessas amostras: fusão e prensagem, a partir do pó do polímero em moldes de aço inoxidável especificamente desenvolvido para esse fim, e torneando-se os discos a partir de cilindros de materiais poliméricos, no caso de alguns polímeros comerciais. Essas amostras foram em seguida recobertas com uma fina película de grafita coloidal, para aumentar a sua emissividade. Em alguns casos houve a necessidade de se recobrir a amostra com uma finíssima camada de ouro antes da aplicação da camada de grafita coloidal. Isto foi necessário para se impedir que o feixe de laser incidente na face frontal da amostra viesse a atingir o detector devido a transparência de alguns materiais.

Utilizando o procedimento descrito anteriormente, foi então, medida a difusividade térmica dos materiais selecionados a temperatura ambiente e a $100{ }^{\circ} \mathrm{C}$. Em cada temperatura foram feitas de 10 a 15 medidas, considerando-se a média aritmética entre as mesmas. Os resultados obtidos são mostrados na tabela 1. Conhecendo-se o calor específico e a densidade do material pode-se, então, calcular a sua condutividade térmica, utilizando-se para isso a equação 4 .

\section{Resultados e Discussão}

A reprodutibilidade dos resultados experimentais obtidos foi excelente. Os valores de difusividade apresentados na 
Tabela 1. Valores da difusividade térmica obtidos para as várias amostras em duas temperaturas.

\begin{tabular}{|c|c|c|c|}
\hline \multirow{2}{*}{ Amostra } & \multirow{2}{*}{ Material } & \multicolumn{2}{|c|}{$\begin{array}{c}\text { Difusividade } \\
\text { térmica }\left(\mathbf{x 1 0}^{-6} \mathrm{~m}^{2} / \mathrm{s}\right)\end{array}$} \\
\hline & & $\mathrm{T}=25^{\circ} \mathrm{C}$ & $\mathrm{T}=100{ }^{\circ} \mathrm{C}$ \\
\hline A1 & Polipropileno & 0,1275 & 0,0981 \\
\hline A2 & Nylon & 0,1466 & 0,1088 \\
\hline A3 & $\begin{array}{c}\text { Poli(cloreto de vinilideno) } \\
\text { PVC rígido }\end{array}$ & 0,1361 & 0,0939 \\
\hline A4 & $\begin{array}{l}\text { Poli(fluoreto de vinilideno) } \\
\text { PVDF }\end{array}$ & 0,1115 & 0,0633 \\
\hline A5 & $\begin{array}{l}\text { Copolímero P(VDF-TrFE) } \\
\text { com } 80 \% \text { em mol de VDF }\end{array}$ & 0,0934 & 0,0695 \\
\hline A6 & $\begin{array}{l}\text { Copolímero P(VDF-TrFE) } \\
\text { com } 70 \% \text { em mol de VDF }\end{array}$ & 0,0797 & 0,0628 \\
\hline A7 & $\begin{array}{c}\text { Blenda PVDF/POMA } \\
\text { com } 1 \% \text { em peso de POMA }\end{array}$ & 0,1033 & 0,0748 \\
\hline A8 & $\begin{array}{c}\text { Blenda PVDF/POMA } \\
\text { com } 2 \% \text { em peso de POMA }\end{array}$ & 0,1505 & 0,1127 \\
\hline A9 & $\begin{array}{c}\text { Blenda PVDF/ P(VDF-TrFE), } \\
70 \% \text { em peso de PVDF }\end{array}$ & 0,0943 & 0,0728 \\
\hline A10 & $\begin{array}{c}\text { Blenda PVDF/ P(VDF-TrFE), } \\
50 \% \text { em peso de PVDF }\end{array}$ & 0,1029 & 0,0758 \\
\hline
\end{tabular}

Tabela 1 para cada material, em cada uma das temperaturas de ensaio, é o valor médio calculado a partir de quinze medidas experimentais feitas a temperatura ambiente $\left(25^{\circ} \mathrm{C}\right) \mathrm{e}$ dez medidas feitas a $100^{\circ} \mathrm{C}$. A Tabela 2 mostra os desvios percentuais entre os valores mínimo e médio $\left(\delta_{\min }\right)$, e entre os valores máximo e médio $\left(\delta_{\max }\right)$ das difusividades térmicas obtidas experimentalmente. Dispersão entre os valores obtidos em baixas temperaturas, principalmente à temperatura ambiente, é sempre esperada, uma vez que a radiação ambiente também é detectada pelo fotodetector, exercendo assim uma certa influência no resultado experimental em baixas temperaturas. Par minimizar este problema, foram feitas mais medidas a temperatura ambiente que a $100^{\circ} \mathrm{C}$, considerando-se a seguir a média aritmética entre elas.

A Tabela 3 mostra os resultados experimentais obtidos neste trabalho e alguns valores de difusividade térmica encontrados na literatura.

Devido à escassez de dados de difusividade térmica de polímeros na literatura só foram encontrados valores para as

Tabela 2. Desvios percentuais entre os valores mínimo e médio $\left(\delta_{\text {min }}\right)$ e entre os valores máximo e médio $\left(\delta_{\max }\right.$ das difusividades térmicas medidas experimentalmente.

\begin{tabular}{cccccccccccc}
\hline $\mathbf{T}\left({ }^{\circ} \mathbf{C}\right)$ & & A1 & A2 & A3 & A4 & A5 & A6 & A7 & A8 & A9 & A10 \\
\hline \multirow{2}{*}{25} & $\delta \sin$ & 4 & 14 & 6 & 9 & 8 & 4 & 8 & 8 & 7 & 6 \\
\cline { 3 - 11 } & $\delta \max$ & 3 & 10 & 7 & 5 & 5 & 2 & 2 & 8 & 8 & 6 \\
\hline \multirow{2}{*}{100} & $\delta \min$ & 4 & 4 & 7 & 2 & 2 & 3 & 3 & 4 & 5 & 5 \\
\cline { 3 - 11 } & $\delta \operatorname{smax}$ & 5 & 6 & 7 & 3 & 2 & 4 & 2 & 5 & 5 & 6 \\
\hline
\end{tabular}

Tabela 3. Comparação entre os resultados obtidos experimentalmente e resultados da literatura.

\begin{tabular}{|c|c|c|c|c|}
\hline \multirow{3}{*}{ Amostra } & \multicolumn{4}{|c|}{ Difusividade térmica $\left(\times 10^{-6} \mathrm{~m}^{2} / \mathrm{s}\right)$} \\
\hline & \multicolumn{2}{|c|}{$\begin{array}{c}\text { Técnica de pulso } \\
\text { de energia }\end{array}$} & \multicolumn{2}{|c|}{ Literatura } \\
\hline & $\mathrm{T}=25^{\circ} \mathrm{C}$ & $\mathrm{T}=100{ }^{\circ} \mathrm{C}$ & $\mathrm{T}=25^{\circ} \mathrm{C}$ & $\mathrm{T}=100{ }^{\circ} \mathrm{C}$ \\
\hline A1 & 0,1275 & 0,0981 & $0,150^{[7]}$ e $0,090^{[2]}$ & $0,064^{[7]}$ \\
\hline A2 & 0,1466 & 0,1088 & $\begin{array}{c}0,130^{[7]} \text { e } 0,101^{[8]} \\
0,130^{[2]}\end{array}$ & - \\
\hline $\mathrm{A} 3$ & 0,1361 & 0,0939 & $\begin{array}{l}0,1167^{[7]} \\
0,1100^{[2]}\end{array}$ & $0,0814^{[7]}$ \\
\hline
\end{tabular}

Tabela 4. Comparação entre os resultados obtidos pela técnica de fio quente e técnica de pulso de energia.

\begin{tabular}{|c|c|c|c|}
\hline \multirow{2}{*}{ Material } & \multicolumn{3}{|c|}{$\begin{array}{l}\text { Difusividade térmica } \\
\qquad\left(\times 10^{-6} \mathrm{~m} 2 / \mathrm{s}\right)\end{array}$} \\
\hline & $\begin{array}{l}\text { Técnica de } \\
\text { fio quente }\end{array}$ & $\begin{array}{c}\text { Técnica de } \\
\text { pulso de energia }\end{array}$ & $\begin{array}{c}\text { Desvio } \\
\text { percentual }\end{array}$ \\
\hline $\mathrm{PVC}$ & 0,1359 & 0,1361 & 0,1 \\
\hline Nylon & 0,1473 & 0,1466 & 0,5 \\
\hline Polipropileno & 0,1455 & 0,1275 & 14,1 \\
\hline
\end{tabular}

amostras A1, A2 e A3. Normalmente só são encontrados valores para os polímeros de maior comercialização, e ainda assim, os poucos dados existentes às vezes são conflitantes, o que torna mais difícil uma efetiva comparação entre os resultados experimentais obtidos e os dados existentes na literatura.

Para três polímeros comerciais foi possível uma comparação entre os resultados obtidos pelo método do fio quente e pela técnica de pulso de energia, a temperatura ambiente. A tabela 4 mostra essa comparação.

Verifica-se pela Tabela 4 que os resultados obtidos pela técnica de pulso de energia, e pelo método do fio quente, estão em excelente concordância para o PVC e para o Nylon, com um desvio percentual muito pequeno. Já para o polipropileno o desvio foi maior, da ordem de $14 \%$, e pode ser atribuído a história térmica do polímero. Para o PVC e para o Nylon, as amostras para ensaio em ambas as técnicas, foram retiradas da mesma placa do material comercial. No caso do polipropileno, a amostra para medida pelo método do fio quente foi retirada de uma placa comercial, enquanto que a amostra para ensaio pela técnica de pulso de energia foi preparada em laboratório por prensagem a quente. Este fato mostra também a influência da história térmica do polímero nas suas propriedades térmicas. Os resultados apresentados nesta tabela mostram mais uma vez a viabilidade da utilização da técnica de pulso de energia para materiais poliméricos.

\section{Conclusões}

A técnica de pulso de energia, muito utilizada na determinação das propriedades térmicas de materiais cerâmicos e metálicos 
mostrou-se extremamente adequada também para materiais poliméricos. A grande vantagem desta técnica é a utilização de amostras pequenas. Isto torna o processo de medida extremamente rápido, pois o tempo necessário para a amostra atingir a temperatura de ensaio desejada é bastante pequeno. Outra grande vantagem é que, o fato das amostras serem pequenas, viabiliza também a utilização deste método para materiais onde a preparação de amostras maiores torna-se problemática.

\section{Agradecimentos}

Os autores agradecem a FAPESP (Proc. 2003/00294-2) e ao CNPq (Proc. 302969/2002-0) pelo apoio financeiro recebido.

\section{Referências Bibliográficas}

1. Rauwendaal, C.-“Polymer Extrusion”, Hanser Publishers, Carl Hanser Verlag, Munich (1986).
2. Ogorkiewicz, R. M.-"Thermoplastics-Properties and Design", John Wiley \& Sons, London (1974).

3. Santos, W. N. dos \& Gregório Filho, R. - Journal of Applied Polymer Science, 85, p.1779 (2002).

4. Parker, W. J.; Jenkins, R. J.; Butter, C. P. \& Abbot, G. L.J. Appl. Phys., 32, p.1679 (1961).

5. Clark, L. M., \& Taylor, R. E. - J. Appl. Phys., 46, p.714 (1975).

6. Taylor, R. - J. Phys. Sci. Instrum., 13, p.1193 (1980).

7. Touloukian, Y. S.; Powell, R. W. \& Ho, C.Y.- "Thermophysical Properties of Matter”, IFI Plenum, New York (1973).

8. Crawford, R. J.-“Plastics Engineering”, ButterworthHeinemann, Oxford (1998).

Recebido: $15 / 08 / 03$

Aprovado: 15/10/03 